\title{
Molecular cloning and site-directed mutagenesis of leucine-based sorting motifs of the porcine invariant chain
}

\author{
F.Z. Xu' ${ }^{1}$, Y. Dai ${ }^{2}$, L.M. Li ${ }^{1}$, X.L. Ding ${ }^{1}$ and W.Y. Yu ${ }^{1}$ \\ ${ }^{1}$ Anhui Agricultural University, Hefei, China \\ ${ }^{2}$ Institute of Animal Husbandry and Veterinary Science, \\ Anhui Academy of Agricultural Sciences, Hefei, China \\ Corresponding author: L.M. Li \\ E-mail:1lm56@ahau.edu.cn
}

Genet. Mol. Res. 12 (4): 4489-4499 (2013)

Received March 20, 2012

Accepted August 27, 2012

Published January 4, 2013

DOI http://dx.doi.org/10.4238/2013.January.4.10

\begin{abstract}
Invariant chain (Ii) is a transmembrane protein that associates with MHC class II molecules in the endoplasmic reticulum. The cytoplasmic tail of Ii contains two leucine residues able to direct Ii to the endocytic pathway. We obtained the pig Ii gene by RT-PCR. Mutated Ii was prepared via site directed mutagenesis by the PCR Megaprimer method to study the effect of the two leucines on the localization of pig Ii. These mutated fragments were ligated to the vector pmCherry-C1. The recombinant plasmids were transiently transfected into COS7 cells with Lipofectamine ${ }^{\mathrm{TM}}$ 2000. Fluorescence of fusion proteins (mCherry-Ii) was observed with a fluorescent microscope. Amino acid sequence alignment showed that pig Ii has domains similar to those seen in other mammalian Ii, including the cytoplasmic, transmembrane, class II-associated Ii-derived peptide, and trimerization domains. Based on observations with the fluorescent microscope, we found that two leucine-based motifs are required for pig Ii intracellular localization, and that both motifs independently mediate this function in Ii.
\end{abstract}

Key words: Invariant chain; Domain analysis; Localization; CLIP; TRIM 


\section{INTRODUCTION}

The major histocompatibility complex (MHC) class II molecules are heterodimers ( $\alpha$ and $\beta$ chain) that associate in the endoplasmic reticulum with the invariant chain (Ii) (Sung and Jones, 1981), and are then transported by the endocytic pathway where Ii is proteolytically processed and dissociated from the class II molecules (Neefjes et al., 1990; Pieters et al., 1991). Ii is well characterized in humans (O'Sullivan et al., 1987), rodents (Lennon-Dumenil et al., 2001), and poultry (Liu et al., 2011). Alternative initiation of transcription and differential splicing of the transcription products generate four different isoforms in humans (O'Sullivan et al., 1987), but only two isoforms in mice (Lennon-Dumenil et al., 2001) and chicken (Zhong et al., 2006). The major form of Ii (P33) contains four domains: the tail of cytoplasmic, transmembrane domain (TM), class II-associated Ii-derived peptide (CLIP), trimerization (TRIM) and thyroglobulin $(\mathrm{Tg})$ domains.

Ii in different species containing pairs of Leu-Ile and Met-Leu or Val-Leu in their cytosolic tails is conserved (Pieters et al., 1993; Bremnes et al., 1994; Sandoval et al., 1994). Ii directs associated class II molecules to the endocytic route (Bakke and Dobberstein, 1990; Lotteau et al., 1990; Busch et al., 2005; Holst et al., 2008; Xu et al., 2008) by two independent leucine-based sorting signals in the amino-terminal cytoplasmic tail (Bremnes et al., 1994). Alanine-scanning mutagenesis demonstrated differential requirements for clathrin-associated adaptor protein complexes AP1 and AP2 binding to Ii at the level of residues around the critical leucine residues (Kongsvik et al., 2002).

The cytosolic tail of pig Ii was found to contain pairs of Leu-Ile and Met-Leu. However, the functional properties of the two leucine motifs are so far unclear in pig Ii. In this report, we cloned the Ii gene using RT-PCR from pig spleen and applied bioinformatic tools to analyze its predicted amino acid domains and protein structure. Furthermore, the mutations were obtained via site-directed mutagenesis by the PCR megaprimer method to study the effect of both leucines on the localization of pig Ii. Thus, after validating the feasibility of the experimental system, we investigated the roles of two leucine residues within the cytoplasmic tail of pig Ii in Ii-induced endosomal vacuolization, or Ii intracellular localization. Here, we applied mCherry-fused Ii to definitively detect two leucine residues mediating Ii endosomal targeting by the direct fluorescence microscope. It was demonstrated that the localization of Ii-induced intracellular requires di-Leu motifs.

\section{MATERIAL AND METHODS}

\section{Expression vector}

pmCherry-C1 (Clontech, Mountain View, CA, USA) is a mammalian expression vector designed to express a protein of interest fused to the C-terminus of mCherry, a mutant fluorescent protein derived from the tetrameric Discosoma sp red fluorescent protein, DsRed (Shaner et al., 2004). The pmCherry-C1 vector can be transfected into mammalian cells using any standard transfection method. A Kozak (1987) consensus sequence is located immediately upstream of the pmCherry gene to enhance translational efficiency in eukaryotic systems. The pmCherry-C1 can also be used as a cotransfection marker, as the gene will express mCherry in mammalian cells. 


\section{Cells and cell culture}

COS-7 cells derived from CV1 cells were transformed with an origin-defective mutant of SV40 coding for the wild-type T-antigen (Gluzman, 1981). The cell lines were maintained in RPMI 1640 medium supplemented with $10 \%(\mathrm{v} / \mathrm{v})$ fetal bovine serum (Gibco, USA) and $1 \%$ penicillin/streptomycin at $37^{\circ} \mathrm{C}$ in $5 \% \mathrm{CO}_{2}$.

\section{RT-PCR analysis}

Total RNA was extracted from blood lymphocytes of pig using the Trizol kit (Gibco) following manufacturer instructions. The pig Ii gene was amplified by primers (5'-ATGCGGGTCCCCCGGCCT-3', 5'-GCTCATATCCTAAGATTCAGCA-3') with a onestep RT-PCR kit (Gibco).The PCR products were analyzed by 1.5\% agarose gel electrophoresis and cloned into the PMD18-T vector (Takara, Dalian, China). The identity of Ii was confirmed by sequencing using the ABI Prism automated sequencing method and named PMD18-Ii.

\section{Functional domain analysis of pig Ii}

Sequence alignment was performed with the Align software at the Clustal W network server (http://www.ebi.ac.uk/clustalw/).

\section{Construction of Ii wild-type and mutant expression vector}

PCR primer pair (5'-GAAGATCTATGCGGGTCCCCCGGCCT-3', 5'-GCGTCGAC TCATATCCTAAGATTCAGCA-3') was used to construct the pmCherry-C1-Ii vector. The underlined sequences are $B g l \mathrm{II}$ and SalI restriction enzyme sites, respectively. Point mutations at leucine residues ( $\mathrm{Leu}^{7}$ and $\mathrm{Leu}^{17}$ ) in the cytoplasmic tail of Ii were introduced by PCR-based megaprimer mutagenesis using the recombinant plasmid PMD18-Ii as template. The primers are listed in Table 1 . A volume of $100 \mu \mathrm{L}$ was amplified with the mutated primers and the primer of Ii-F at low annealing temperature. PCR-based megaprimer mutagenesis was used, and the temperature profile was set as follows: 1 cycle of $94^{\circ} \mathrm{C}$ for $5 \mathrm{~min}, 45^{\circ} \mathrm{C}$ for $1 \mathrm{~min}$, and $72^{\circ} \mathrm{C} 1$ for min; 24 cycles of $94^{\circ} \mathrm{C}$ for $40 \mathrm{~s}, 45^{\circ} \mathrm{C}$ for $1 \mathrm{~min}$, and $72^{\circ} \mathrm{C}$ for $1 \mathrm{~min}$; and 1 cycle of $94^{\circ} \mathrm{C}$ for $40 \mathrm{~s}, 45^{\circ} \mathrm{C}$ for $1 \mathrm{~min}$, and $72^{\circ} \mathrm{C}$ for $5 \mathrm{~min}$. After the forward round PCR ended, the high annealing temperature primer of Ii-R was added and temperature profile was set as follows: 25 cycles of $94^{\circ} \mathrm{C}$ for $40 \mathrm{~s}$, $72^{\circ} \mathrm{C}$ for $90 \mathrm{~s}$, and 1 cycle of $72^{\circ} \mathrm{C}$ for $5 \mathrm{~min}$. The final PCR products were cloned into pmCherry-C1 and verified by DNA sequencing.

\section{Transient transfection of COS-7 cells}

COS-7 cell lines were maintained in RPMI 1640 medium supplemented with $10 \%$ fetal bovine serum (Gibco). Briefly, $70 \%$ confluent COS-7 cells were split into 24 wells (with coverslips for immunofluorescence microscopy) 1 day before transfection. COS-7 cells were transfected with Lipofectamine ${ }^{\mathrm{TM}} 2000$ (Gibco), following manufacturer instructions. 
Table 1. Primers for amplification of the Ii mutant.

\begin{tabular}{ll}
\hline Primer & Sequence $\left(5^{\prime} \rightarrow 3^{\prime}\right)$ \\
\hline L7A-R & ATGGTTCCAGATGGCGTCGCGCTGGTC \\
L17A-R & GGGGCGCTGGCCGCCATGGGCAGCTG \\
Ii-F & GAAGA TCTCGAGCTCAA (low annealing BglII) \\
Ii-R & GCGTCGACTGCTTGGCTTTCACCATGTCCACCCCAGAG (high annealing Sall) \\
\hline $\mathrm{F}=$ forward; $\mathrm{R}=$ reverse &
\end{tabular}

$\mathrm{F}=$ forward $\mathrm{R}=$ reverse.

\section{Fluorescence microscopy}

Expression of the mCherry protein was used as a marker of positively transfected cells. At 24 to $48 \mathrm{~h}$ after transfection, cells on coverslips were fixed with $4 \%$ paraformaldehyde and visualized with an Olympus fluorescence microscope (Olympus, Tokyo, Japan).

\section{Results}

\section{Amplification of the Ii gene}

The pig Ii cDNA was obtained and contained a 645-bp open reading frame encoding a putative 215-amino acid protein (Figure 1). The putative amino acid sequence showed a $99 \%$ similarity to the previously identified pig Ii sequence, as determined by BLAST searches. The Ii gene was inserted into pmCherry-C1 and named wild type (WT).

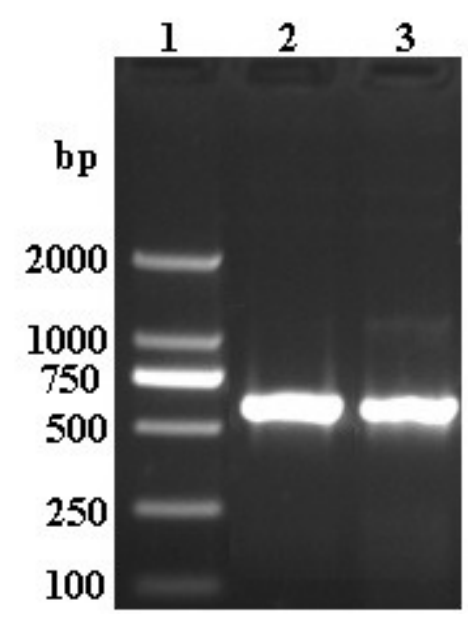

Figure 1. Amplified product of the Ii gene. Lane $1=$ DL2000 markers; lanes 2 and $3=$ amplified PCR products.

\section{Alignment of pig Ii with the Ii of other species}

Pig Ii amino acid sequence was aligned with that of other species. The result showed that among these aligned species, the most highly conserved domains were the TM and Tg, followed by CLIP, cytoplasmic and TRIM domains (Figure 2). 
Cytophenic domin TH domin

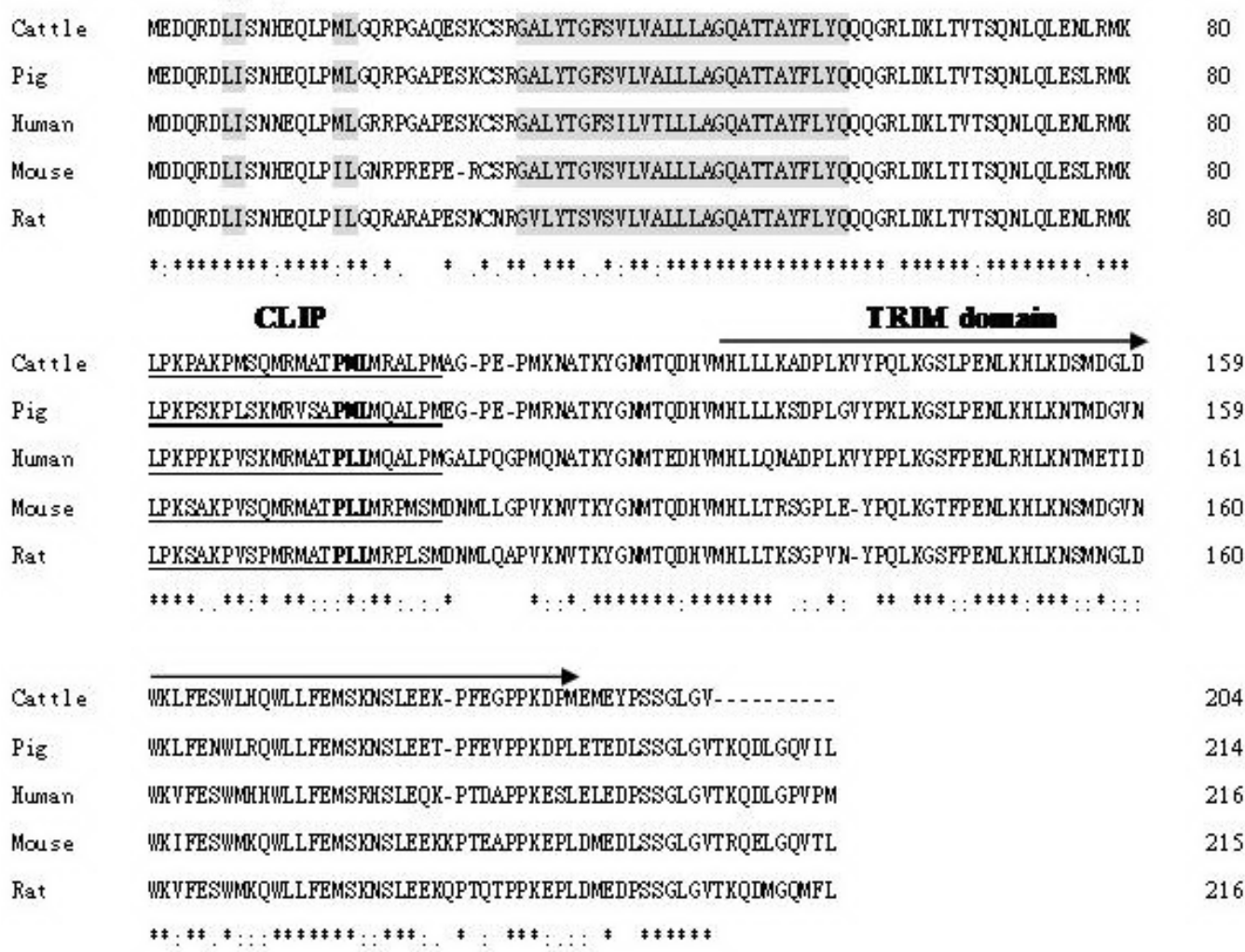

Figure 2. Alignment of pig Ii cDNA with Ii of other species. Alignment of the predicted pig Ii translation product with Ii from cattle (GenBank accession No. BC102388.1), human (GenBank accession No. X00497.1), mouse (GenBank accession No. BC096435.1), and rat (GenBank accession No. BC059152.1). Identical (asterisks) and similar (colons) amino acid residues are indicated. The pairs of Leu-Ile and Met-Leu in the cytosolic tails are conserved. The regions with highest homology are the TM (transmembrane) domains. The CLIP domain (class II-associated invariant chain peptide) is indicated by a single underline. The TRIM (trimerization) domain is indicated by arrows.

\section{The mutated Ii gene}

The mutants were obtained by two times PCR-based point mutated megaprimer. A segment of 120 nucleotides was obtained by the first round of PCR with mutant primers and Ii-F as primers, then by the second round of PCR, and the final 645 nucleotides were amplified with the former segment and Ii-R as primer (Figure 3). The final PCR products were inserted into pmCherry-C1, respectively. The resultant plasmids were named M1-M3.

\section{Effect of two leucine motifs on pig Ii intracellular localization}

To validate that effect of two leucine motifs on Ii-induced endosomal vacuolization, we detected the intracellular localization of mCherry protein expression to estimate the effect 
of M1-M3 mutation constructs (Table 2). The results in Figure 4 indicated that leucine is a key element of the targeting motif. In the control cells transfected with pmCherry-C1 alone there was a strong fluorescent staining of the plasma membrane in COS-7 (Figure 4, C1). When other constructs were transfected into COS-7 cells, different results were obtained by fluorescence microscopy. Fluorescent staining was found in the intracellular vesicles in the cells transfected by the wild-type Ii construct (Figure 4, WT). A site mutation of Leu ${ }^{7}$ (M1) or Leu ${ }^{17}$ (M2) to Ala did not abolish this endosomal localization (Figure 4, M1-M2). Furthermore, in the mutation of both $\mathrm{Leu}^{7}$ and $\mathrm{Leu}^{17}$, fluorescence studies demonstrated strong plasma membrane staining (Figure 4, M3) and with apparent loss of the ability to internalize to endosome transport. The above events indicated that the pig Ii cytoplasmic tail may contain two independent leucine-based sorting signals.

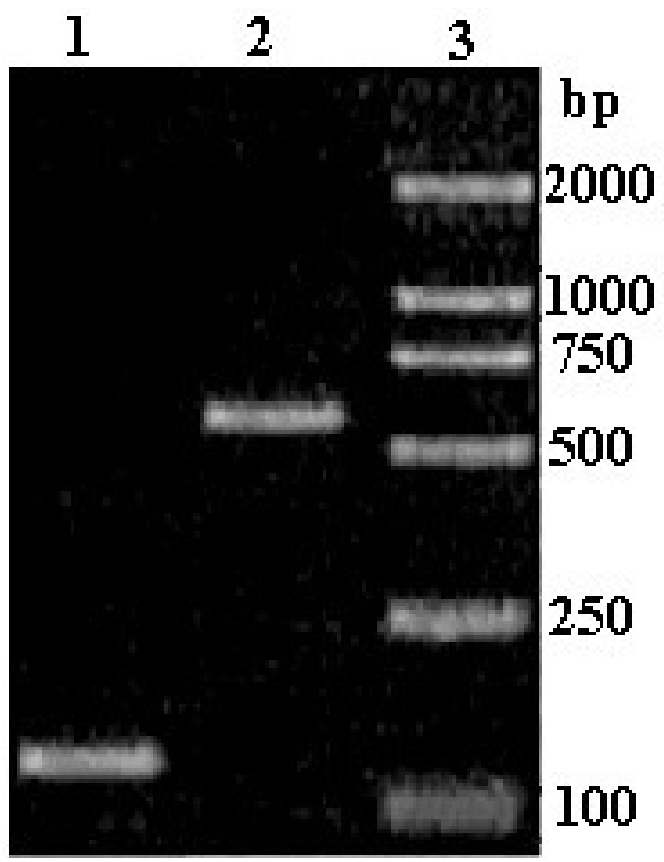

Figure 3. Amplified product of the mutated Ii gene. Lane $1=$ amplified megaprimer by the mutated primer and the low annealing temperature Ii-F primer; lane 2 = amplified PCR product using the megaprimer and the high annealing temperature Ii-R as primer; and lane $3=$ DL2000 markers.

Table 2. Cellular distribution and internalization of the recombinants.

\begin{tabular}{lllc}
\hline No. & Mutation at position & Ii cytoplasmic tail amino acid sequences & Localization \\
\hline WT & Wild type & MEDQRDLISNHEQLPMLGQ--- & V \\
M1 & L7A & MEDQRDAISNHEQLPMLGQ--- & V \\
M2 & L17 A & MEDQRDLISNHEQLPMAGGQ-- & V \\
M3 & L7A, L17A & MEDQRDAISNHEQLPMEGGQ-- & PM \\
\hline
\end{tabular}

Mutations of amino acids to alanine are indicated by "A" in bold and underlined. Mutations of amino acids to leucine are indicated by "L" in bold. Localization by vesicular staining or plasma membrane is indicated by V and PM, respectively. 

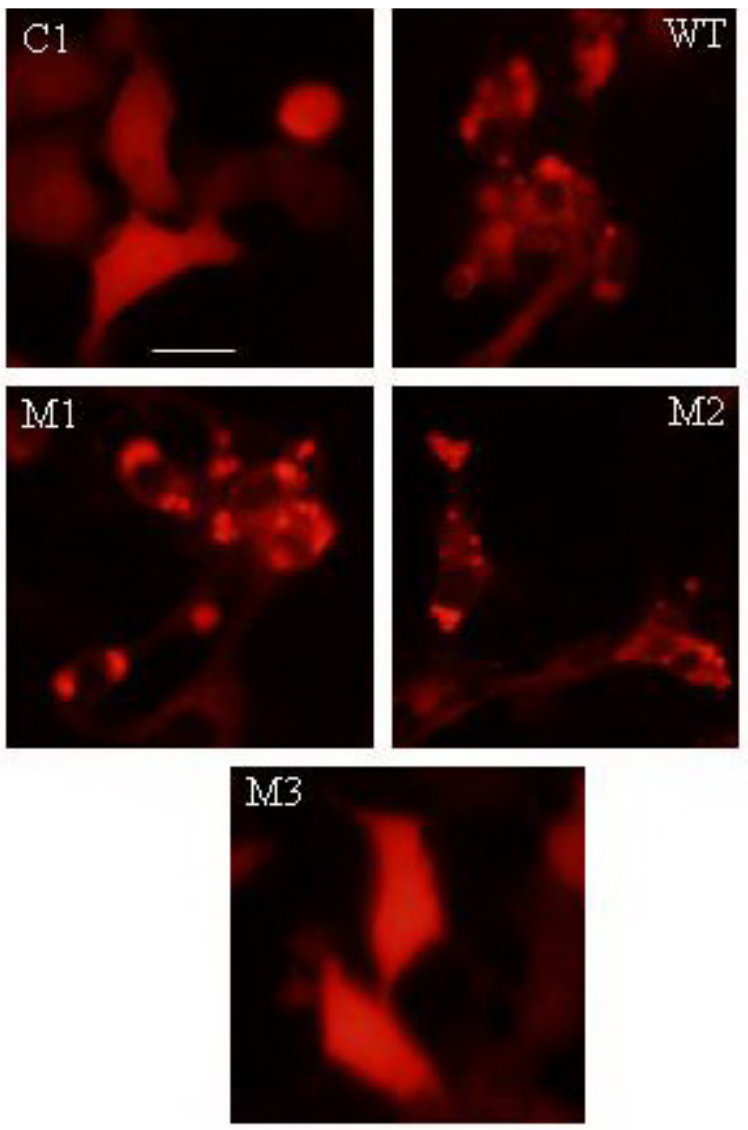

Figure 4. Localization of Ii wild types and mutants in COS-7 cells. COS-7 cells transiently transfected with pmCherry-C1 (C1), wild-type Ii (WT), L7A (M1), L17A (M2), L7A, L17A (M3) were visualized by fluorescence microscopy. Bar $=10 \mu \mathrm{m}$.

\section{DISCUSSION}

\section{Conserved amino acid residues at critical sites are essential for ensuring Ii structure and functions}

In this study, we determined the pig Ii gene by RT-PCR technology. The amino acid sequence analysis indicated that the pig Ii protein showed high similarity in amino acid sequence with other Ii species, with a similar format of domain structure of Ii among various species, the cytoplasmic, TM, CLIP, and TRIM domains (Figure 2).

The cytoplasmic domain of pig Ii contains a highly conserved di-leucine sequence (LeuIle and Met-Leu or Val-Leu), which, like its mammalian counterpart, may play a role in targeting endosomes (Johnson et al., 2001; Silva and Edwards, 2009). The fact that the di-leucine sequence is also highly conserved in other species (rat and mouse) and poultry species (chicken) suggests that this sequence may be phylogenetically conserved. At the amino-terminus, there is 
a highly conserved TM domain consisting of 27 amino acid residues and 23 of them are identical or similar between the aligned species (Figure 2). Ashman and Miller (1999) reported that the TM domain plays a key role in the self-assembly of Ii into a trimer, the formation of which is the basis for the interaction between MHC class II molecules and Ii. The CLIP domain between amino acids 82 and 107 is essential for binding to the class II molecules (Denzin and Cresswell, 1995; Ghosh et al., 1995; Morkowski et al., 1995). The CLIP domain reveals slight diversity and maintains over 50\% identity (Liu et al., 2011). Association with MHC class II related HLA-DM is critical for the removal of CLIP and loading of peptide (Rudensky et al., 1994). CLIP directly occupies the groove of the MHC class II molecule peptide binding region (Gregers et al., 2003), which is the most polymorphic region in all known molecules (Silva and Edwards, 2009). This diversity of MHC class II is believed to be adapted to the diversity of pathogens (Bernatchez and Landry, 2003; Surridge et al., 2008; Meyer-Lucht et al., 2010). Therefore, CLIP may be modified to fit the variant MHC class II under selective pressure.

\section{mCherry protein as the marker of localization in vitro}

When a protein is tagged by fusion to a fluorescent protein, interactions between fluorescent proteins can undesirably disturb targeting or function. Red fluorescent protein, as a marker for gene expression, can monitor target gene expression and protein localization in live cells (Fradkov et al., 2000; Yanushevich et al., 2002). Unfortunately, all wild-type yellow-tored fluorescent proteins reported so far are obligately tetrameric and often toxic or disruptive. The mCherry protein is a red fluorescent protein, derived from the Discosoma sp fluorescent protein "DsRed" by directed evolution first to increase the speed of maturation, then to break each subunit interface while restoring (Shaner et al., 2004). Expression of fusion proteins that retain the fluorescent properties of the unmodified mCherry protein can be monitored by flow cytometry and their localization in vitro can be determined by fluorescent microscopy.

\section{Two leucine-based motifs are endosomal targeting signal}

It has been shown that signals within the cytoplasmic tail of Ii are responsible for the transport of Ii alone and in complex with MHC class II to the endocytic pathway (Bakke and Dobberstein, 1990; Roche et al., 1992; Simonsen et al., 1993). Experiments have also shown that two leucines located at the seven and the seventeenth amino acid residues are sorting signals, and that only one of them can maintain the basic intracellular localization of the pig Ii molecule. The LL/LI signal is present in various molecules, such as the tyrosine kinase receptors (Hanks et al., 1988), in addition to the T-cell receptor (Letourneur and Klausner, 1992) and the two M6PR (Johnson and Kornfeld, 1992). These results are in agreement with a previous observation about leucine-based targeting motifs in mammals and poultry (Bremnes et al., 1994, 2000; Xu et al., 2008).

Nilsson et al. (1991) suggested that the cytoplasmic Ii tail is exchanged with that of galactosyltransferase, which contains a di-leucine, and the resulting molecule is sorted to endosomes. One putative function of the di-leucine signal may be to sort molecules to the endocytic pathway for degradation if they are missorted to the plasma membrane and possibly TGN (Bremnes et al., 1994). Two internalization signals are present within the cytoplasmic tail of Ii, and both of them seem to mediate efficient internalization. In accordance with an 
extrapolation of spatial structure (Figure 5) based on amino acid sequence of pig Ii, which is predicted by software (http://zhanglab.ccmb.med.umich.edu/I-TASSER/output/S96721/), there may be more helices within the pig Ii molecule, and the first $\alpha$-helix would contact the other $\beta$-helices with a hydrophobic structure. The hydrophobic residue $\mathrm{Leu}^{7}$ is located just at the interface of the composed $\alpha$-helix. Also, there may be a long loop at the cytoplasmic side of the membrane, in which $\mathrm{Leu}^{17}$ is situated within the segment of this loop. For all of them, a mutation of Leu ${ }^{7}$ or Leu ${ }^{17}$ could change the first helix or the loop, but that is not enough to alter the whole spatial structure and thereby to disrupt the localization of pig Ii.

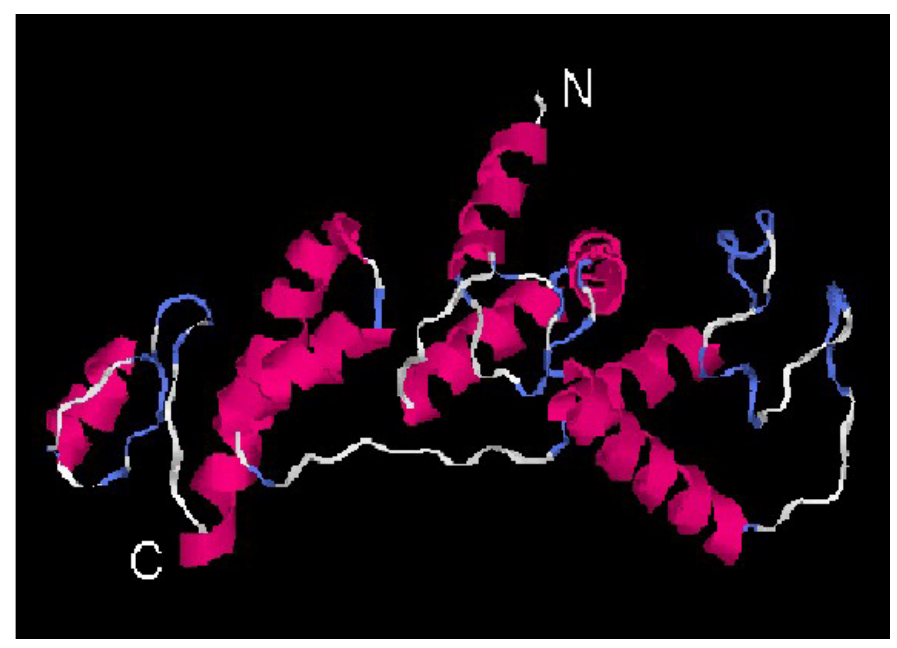

Figure 5. Predicted structure of pig Ii.

\section{CONCLUSION}

We cloned a 645-bp cDNA sequence of pig Ii encoding a protein of 215 amino acids. The pig Ii shows high conservation compared with Ii of other species in major functional domains. Two leucine-based motifs located in the cytoplasmic tail of Ii are required for pig Ii intracellular localization, and both motifs independently mediate this function of Ii.

\section{ACKNOWLEDGMENTS}

Research supported by the National Natural Science Foundation of China (\#31101797) and the College Science Research Project of Anhui Province (\#KJ2011A107).

\section{REFERENCES}

Ashman JB and Miller J (1999). A role for the transmembrane domain in the trimerization of the MHC class II-associated invariant chain. J. Immunol. 163: 2704-2712.

Bakke O and Dobberstein B (1990). MHC class II-associated invariant chain contains a sorting signal for endosomal compartments. Cell 63: 707-716.

Bernatchez L and Landry C (2003). MHC studies in nonmodel vertebrates: what have we learned about natural selection 
in 15 years? J. Evol. Biol. 16: 363-377.

Bremnes B, Madsen T, Gedde-Dahl M and Bakke O (1994). An LI and ML motif in the cytoplasmic tail of the MHCassociated invariant chain mediate rapid internalization. J. Cell Sci. 107: 2021-2032.

Bremnes B, Rode M, Gedde-Dahl M, Nordeng TW, et al. (2000). The MHC class II-associated chicken invariant chain shares functional properties with its mammalian homologs. Exp. Cell Res. 259: 360-369.

Busch R, Rinderknecht CH, Roh S, Lee AW, et al. (2005). Achieving stability through editing and chaperoning: regulation of MHC class II peptide binding and expression. Immunol. Rev. 207: 242-260.

Denzin LK and Cresswell P (1995). HLA-DM induces CLIP dissociation from MHC class II alpha beta dimers and facilitates peptide loading. Cell 82: 155-165.

Fradkov AF, Chen Y, Ding L, Barsova EV, et al. (2000). Novel fluorescent protein from Discosoma coral and its mutants possesses a unique far-red fluorescence. FEBS Lett. 479: 127-130.

Ghosh P, Amaya M, Mellins E and Wiley DC (1995). The structure of an intermediate in class II MHC maturation: CLIP bound to HLA-DR3. Nature 378: 457-462.

Gluzman Y (1981). SV40-transformed simian cells support the replication of early SV40 mutants. Cell 23: 175-182.

Gregers TF, Fleckenstein B, Vartdal F, Roepstorff P, et al. (2003). MHC class II loading of high or low affinity peptides directed by Ii/peptide fusion constructs: implications for T cell activation. Int. Immunol. 15: 1291-1299.

Hanks SK, Quinn AM and Hunter T (1988). The protein kinase family: conserved features and deduced phylogeny of the catalytic domains. Science 241: 42-52.

Holst PJ, Sorensen MR, Mandrup Jensen CM, Orskov C, et al. (2008). MHC class II-associated invariant chain linkage of antigen dramatically improves cell-mediated immunity induced by adenovirus vaccines. J. Immunol. 180: 33393346.

Johnson AO, Lampson MA and McGraw TE (2001). A di-leucine sequence and a cluster of acidic amino acids are required for dynamic retention in the endosomal recycling compartment of fibroblasts. Mol. Biol. Cell 12: 367-381.

Johnson KF and Kornfeld S (1992). A His-Leu-Leu sequence near the carboxyl terminus of the cytoplasmic domain of the cation-dependent mannose 6-phosphate receptor is necessary for the lysosomal enzyme sorting function. J. Biol. Chem. 267: 17110-17115.

Kongsvik TL, Honing S, Bakke O and Rodionov DG (2002). Mechanism of interaction between leucine-based sorting signals from the invariant chain and clathrin-associated adaptor protein complexes AP1 and AP2. J. Biol. Chem. 277: 16484-16488.

Kozak M (1987). An analysis of 5'-noncoding sequences from 699 vertebrate messenger RNAs. Nucleic Acids Res. 15: $8125-8148$.

Lennon-Dumenil AM, Roberts RA, Valentijn K, Driessen C, et al. (2001). The p41 isoform of invariant chain is a chaperone for cathepsin L. EMBO J. 20: 4055-4064.

Letourneur F and Klausner RD (1992). A novel di-leucine motif and a tyrosine-based motif independently mediate lysosomal targeting and endocytosis of CD3 chains. Cell 69: 1143-1157.

Liu SJ, Chen FF, Dai Y, Wu C, et al. (2011). Molecular characterization and tissue-specific expression of invariant chain in the muscovy duck (Cairina moschata). Genet. Mol. Res. 10: 2867-2880.

Lotteau V, Teyton L, Peleraux A, Nilsson T, et al. (1990). Intracellular transport of class II MHC molecules directed by invariant chain. Nature 348: 600-605.

Meyer-Lucht Y, Otten C, Püttker T, Pardini R, et al. (2010). Variety matters: adaptive genetic diversity and parasite load in two mouse opossums from the Brazilian Atlantic forest. Conserv. Genet. 11: 2001-2013.

Morkowski S, Goldrath AW, Eastman S, Ramachandra L, et al. (1995). T cell recognition of major histocompatibility complex class II complexes with invariant chain processing intermediates. J. Exp. Med. 182: 1403-1413.

Neefjes JJ, Stollorz V, Peters PJ, Geuze HJ, et al. (1990). The biosynthetic pathway of MHC class II but not class I molecules intersects the endocytic route. Cell 61: 171-183.

Nilsson T, Lucocq JM, Mackay D and Warren G (1991). The membrane spanning domain of $\beta$-1,4-galactosyltransferase specifies trans Golgi localization. EMBO J. 10: 3567-3575.

O'Sullivan DM, Noonan D and Quaranta V (1987). Four Ia invariant chain forms derive from a single gene by alternate splicing and alternate initiation of transcription/translation. J. Exp. Med. 166: 444-460.

Pieters J, Horstmann H, Bakke O, Griffiths G, et al. (1991). Intracellular transport and localization of major histocompatibility complex class II molecules and associated invariant chain. J. Cell Biol. 115: 1213-1223.

Pieters J, Bakke O and Dobberstein B (1993). The MHC class II-associated invariant chain contains two endosomal targeting signals within its cytoplasmic tail. J. Cell Sci. 106: 831-846.

Roche PA, Teletski CL, Karp DR, Pinet V, et al. (1992). Stable surface expression of invariant chain prevents peptide presentation by HLA-DR. EMBO J. 11: 2841-2847.

Rudensky AY, Maric M, Eastman S, Shoemaker L, et al. (1994). Intracellular assembly and transport of endogenous 
peptide-MHC class II complexes. Immunity 1: 585-594.

Sandoval IV, Arredondo JJ, Alcalde J, Gonzalez Noriega A, et al. (1994). The residues Leu(Ile)475-Ile(Leu, Val, Ala)476, contained in the extended carboxyl cytoplasmic tail, are critical for targeting of the resident lysosomal membrane protein LIMP II to lysosomes. J. Biol. Chem. 269: 6622-6631.

Shaner NC, Campbell RE, Steinbach PA, Giepmans BN, et al. (2004). Improved monomeric red, orange and yellow fluorescent proteins derived from Discosoma sp. red fluorescent protein. Nat. Biotechnol. 22: 1567-1572.

Silva MC and Edwards SV (2009). Structure and evolution of a new avian MHC class II B gene in a sub-Antarctic seabird, the thin-billed prion (Procellariiformes: Pachyptila belcheri). J. Mol. Evol. 68: 279-291.

Simonsen A, Momburg F, Drexler J, Hammerling GJ, et al. (1993). Intracellular distribution of the MHC class II molecules and the associated invariant chain (Ii) in different cell lines. Int. Immunol. 5: 903-917.

Sung E and Jones PP (1981). The invariant chain of murine Ia antigens: its glycosylation, abundance and subcellular localization. Mol. Immunol. 18: 899-913.

Surridge AK, van der Loo W, Abrantes J, Carneiro M, et al. (2008). Diversity and evolutionary history of the MHC DQA gene in leporids. Immunogenetics 60: 515-525.

Xu FZ, Ye H, Wang JJ and Yu WY (2008). The effect of site-directed mutagenesis of the ambient amino acids of leucinebased sorting motifs on the localization of chicken invariant chain. Poult. Sci. 87: 1980-1986.

Yanushevich YG, Staroverov DB, Savitsky AP, Fradkov AF, et al. (2002). A strategy for the generation of non-aggregating mutants of Anthozoa fluorescent proteins. FEBS Lett. 511: 11-14.

Zhong D, Yu W, Bao M, Xu Z, et al. (2006). Molecular cloning and mRNA expression of duck invariant chain. Vet. Immunol. Immunopathol. 110: 293-302. 